\title{
Desarrollo de Zeolitas Ga-ZSM-11 para la Síntesis de Ácido Láctico
}

\section{Development of Ga-ZSM-11 zeolites for the synthesis of lactic acid}

Presentación: 06/10/2020

\author{
Doctorando: \\ Emilce Daniela Galarza \\ Grupo Zeolitas, Centro de Investigación y Tecnología Química (CITeQ)- Facultad Regional Córdoba, Universidad \\ Tecnológica Nacional, Argentina \\ egalarza@frc.utn.edu.ar, emilce.galarza@gmail.com
}

\section{Directora:}

\section{María Soledad Renzini}

\section{Co-directora:}

\section{Clara Saux}

\section{Resumen}

En el presente trabajo se analiza la influencia de la temperatura de reacción y del contenido de galio en la zeolita ZSM-11 (estructura MEL) en la obtención de ácido láctico a partir de dihidroxiacetona (DHA). El catión metálico fue incorporado por impregnación húmeda en distintas concentraciones ( 2-10\% p/p). La estructura ZSM-11 se confirmó por DRX para todas las muestras, en tanto que en los materiales impregnados la cristalinidad disminuyó a medida que se incrementó la cantidad del catión incorporado al catalizador. Por el método BET se analizó el área superficial y se encontró que la misma disminuye al aumentar el contenido de Ga incorporado. Mediante FTIR con adsorción de piridina se determinó la cantidad, fuerza y relación de sitios ácidos. El estudio por TPR señaló la reducción de las especies superficiales vecinas a los enlaces Ga-O y la reducción de $\mathrm{Ga}^{+3}$ a $\mathrm{Ga}^{+}$. El análisis de reflectancia difusa de UV-visible indicó la dispersión de los posibles aglomerados de óxidos de galio. Dichos materiales exhibieron un buen desempeño catalítico en la conversión de dihidroxiacetona (DHA) a ácido láctico, molécula plataforma de gran interés actual.

Palabras clave: Ga-Zeolita, Síntesis y Caracterización, Acidez de Lewis, Ácido Láctico, Dihidroxiacetona.

\begin{abstract}
In the present work, we analyzed the influence of the reaction temperature and the gallium content in zeolite ZSM11 (MEL structure) in the production of lactic acid from dihydroxyacetone (DHA). The metal cation was incorporated by wet impregnation in different concentrations $(\sim 2-10 \% \mathrm{w} / \mathrm{w})$. The ZSM-11 structure was confirmed by XRD for all samples, whereas in the impregnated materials the crystallinity decreased as the amount of the cation incorporated in the catalyst increased. The surface area was analyzed by BET method and it was found that it decreases when the incorporated Ga content increases. By means of FTIR with pyridine adsorption, the amount, strength and ratio of acidic sites were determined. The study by TPR indicated the reduction of the superficial species neighboring the Ga-O bonds and the reduction of $\mathrm{Ga}^{+3}$ to $\mathrm{Ga}^{+}$. The UV-visible diffuse reflectance analysis indicated the dispersion of the possible agglomerates of gallium oxides. These materials exhibited a good catalytic performance in the conversion of dihydroxyacetone (DHA) to lactic acid, a platform molecule of great current interest.
\end{abstract}

Keywords: Ga-Zeolite, Synthesis and characterization, Lewis acidity, lactic acid, Dihydroxyacetone. 


\section{Introducción}

La producción de productos químicos y materiales a partir de biomasa lignocelulósica atrae un interés sustancial. El ácido láctico se ha identificado como una de las moléculas plataforma más relevantes que conducen a una variedad de productos químicos conocidos, así como a nuevos polímeros biológicos (Ennaert et al., 2016). En la actualidad, la producción industrial de ácido láctico comprende la fermentación microbiana de glucosa o sacarosa. Esta ruta enzimática es de capital intensivo y no es atractiva para el medio ambiente debido a las diversas etapas de separación, neutralización y purificación. Por ello, la catálisis heterogénea cobra relevancia como una ruta más sostenible. La isomerización catalizada por ácido de los compuestos derivados del azúcar C3 dihidroxiacetona (DHA) y gliceraldehído (GLY) representa la alternativa más prometedora. Ambos sustratos de la triosa se pueden obtener a partir del glicerol, que está fácilmente disponible como un subproducto de la fabricación de biocombustibles.

Los catalizadores homogéneos de haluros metálicos (por ejemplo, $\mathrm{SnCl}_{2}, \mathrm{SnCl}_{4} \cdot 5 \mathrm{H}_{2} \mathrm{O}$ ) se investigaron primero para esta reacción, logrando altos rendimientos en unas pocas horas (Hayashi \& Sasaki, 2005).

Sobre la base de estos hallazgos, se desarrollaron con éxito catalizadores heterogéneos eficientes a base de estaño mediante la incorporación de este metal en la estructura de materiales porosos. Sin embargo, dado que la aplicación industrial de materiales a base de Sn podría verse obstaculizada por la escasez de estaño, las zeolitas de aluminosilicato comerciales también se han evaluado para la producción de ácido láctico (Pescarmona et al., 2010). Actualmente ha cobrado interés el uso de galio incorporándose de distintas maneras, tanto durante la síntesis como en tratamientos posteriores (Dapsens et al., 2013, 2014). Por ellos, aquí exponemos la incorporación por impregnación húmeda de distintas concentraciones de galio en la matriz MEL (zeolita ZSM-11) demostrando una alta selectividad en la conversión de DHA a ácido láctico en pocas horas de reacción y a distintas temperaturas. El estudio de caracterización de los catalizadores permitirá correlacionar el efecto de las distintas cargas porcentuales de Ga en las muestras zeolíticas con la actividad catalítica

\section{Desarrollo}

\section{Síntesis de los catalizadores}

Las zeolitas microporosas con estructura ZSM-11 se prepararon por síntesis hidrotérmica empleando aluminato de sodio y sílice como fuentes de aluminio y silicio respectivamente, hidróxido de tetrabutilamonio (TBAOH) como agente director de estructura y agua destilada (Chu, 1973). Se dejó cristalizar en autoclave a $140^{\circ} \mathrm{C}$ y presión autogenerada durante 2 días. El material obtenido fue lavado con agua destilada hasta alcanzar pH neutro y secado en estufa. Posteriormente se desorbió en corriente de $\mathrm{N}_{2}$ y temperatura programada hasta $500^{\circ} \mathrm{C}$ durante $8 \mathrm{~h}$ y finalmente se calcinó a la misma temperatura. Para obtener la forma $\mathrm{NH}_{4}$-zeolita se realizó un intercambio con una solución de cloruro de amonio $\left(\mathrm{NH}_{4} \mathrm{Cl}\right) 1 \mathrm{M}$ a $80^{\circ} \mathrm{C}$. Luego se desorbió en corriente de $\mathrm{N}_{2}$ y calcinó con las condiciones antes descriptas para obtener la forma protónica H ZSM 11.

En la impregnación húmeda (IH) se utilizó la forma $\mathrm{NH}_{4}$-ZSM-11. Se incorporó cantidad suficiente de nitrato de galio $\left(\mathrm{Ga}\left(\mathrm{NO}_{3}\right)_{3}\right)$ para obtener un porcentaje de 2, 5, 7 y 10\% p/p de Ga. La muestra recuperada se desorbió con nitrógeno y se calcinó a $500^{\circ} \mathrm{C}$ por 8 h. Los catalizadores obtenidos se denominaron IH Ga02, IH Ga05, IH Ga07 y IH Ga10.

\section{Caracterización de los catalizadores}

La estructura cristalina de los catalizadores fue determinada por difracción de Rayos X (XRD) en un difractómetro $X$ pert PANanalytical, en un rango de $2 \theta$ entre $5-60^{\circ}$, con un paso de $0.026^{\circ}$, a una velocidad de $5^{\circ} / \mathrm{min}$.

El área superficial de los materiales se determinó por el método Brunauer-Emmet-Teller (BET) en un equipo Pulse Chemisorb de Micromeritics 2700 con absorción de $\mathrm{N}_{2}$ a $77 \mathrm{~K}$.

Los análisis de infrarrojo (FTIR) se realizaron en un espectrómetro Thermo Scientific Nicolet iS10 a los efectos de identificar y cuantificar sitios ácidos del material zeolítico, para tal fin se les adsorbió a las muestras piridina al vacío a temperatura ambiente y desorbió a $400^{\circ} \mathrm{C}$ y $10^{-4}$ Torr, empleando una celda termostatizada con una ventana de CaF2 conectada a una línea de vacío. El número de sitios ácidos de Brönsted y Lewis fue calculado con el área de las bandas de absorción a $1545 \mathrm{~cm}^{-1}$ y $1455 \mathrm{~cm}^{-1}$ respectivamente.

El análisis de reducción a temperatura programada (TPR) se midió en un equipo Pulse Chemisorb 2720 Micromeritics; las muestras fueron calentadas a razón de $10^{\circ} \mathrm{C} / \mathrm{min}$ desde 200 a $800^{\circ} \mathrm{C}$ en presencia de hidrógeno $(5 \%$ $\mathrm{H}_{2} / \mathrm{N}_{2}$ ). La reducción ocurrida se monitoreó por el consumo de $\mathrm{H}_{2}$. 


\section{Actividad catalítica}

La reacción de isomerización de DHA (Aldrich 97\%) a ácido láctico se llevó a cabo en un vial de vidrio de $2 \mathrm{ml}$ a distintas temperaturas $\left(90-110^{\circ} \mathrm{C}\right)$ con presión autogenerada por $4 \mathrm{~h}$ con agitación constante. Se utilizó agua bidestilada como medio de reacción, 16 mg de catalizador y 24 mg de DHA (Dapsens et al., 2014). La concentración del reactivo y productos de reacción fue determinada por Cromatografía Líquida de Alta Resolución (HPLC) en un equipo Jasco UV-975/ PU-980, empleando una columna Aminex HPX - 87H, detector de Índice de Refracción Jasco RI-4030. La fase móvil fue ácido sulfúrico $\left(\mathrm{pH} \mathrm{3,3)} \mathrm{con} \mathrm{un} \mathrm{flujo} \mathrm{de} 0.4 \mathrm{ml} / \mathrm{min}\right.$ y una temperatura de columna de $40^{\circ} \mathrm{C}$. La identificación y cuantificación de los productos de reacción fue realizada por comparación con los estándares puros, mediante curvas de calibración.

\section{Resultados}

\section{Caracterización de los catalizadores}

El análisis por XRD confirma la estructura del tipo ZSM-11 para todos los materiales estudiados (Figura 1). Se observa una disminución de la cristalinidad relativa (Tabla 1), con respecto a la matriz de partida, a medida que aumenta el contenido de galio incorporado. A su vez, comparando los difractogramas con el patrón de difracción del óxido de galio (III) $\left(\mathrm{Ga}_{2} \mathrm{O}_{3}\right)$ no se observa la fase cristalina del óxido. Lo que se puede deber a que las especies Ga se

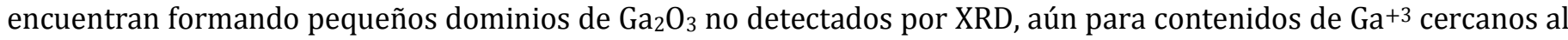
$10 \% \mathrm{p} / \mathrm{p}$.

Los valores de área superficial BET (Tabla 1) decrecen también con el aumento del contenido de galio. Dicho comportamiento puede deberse al bloqueo de poros producido por las especies de Ga incorporadas.

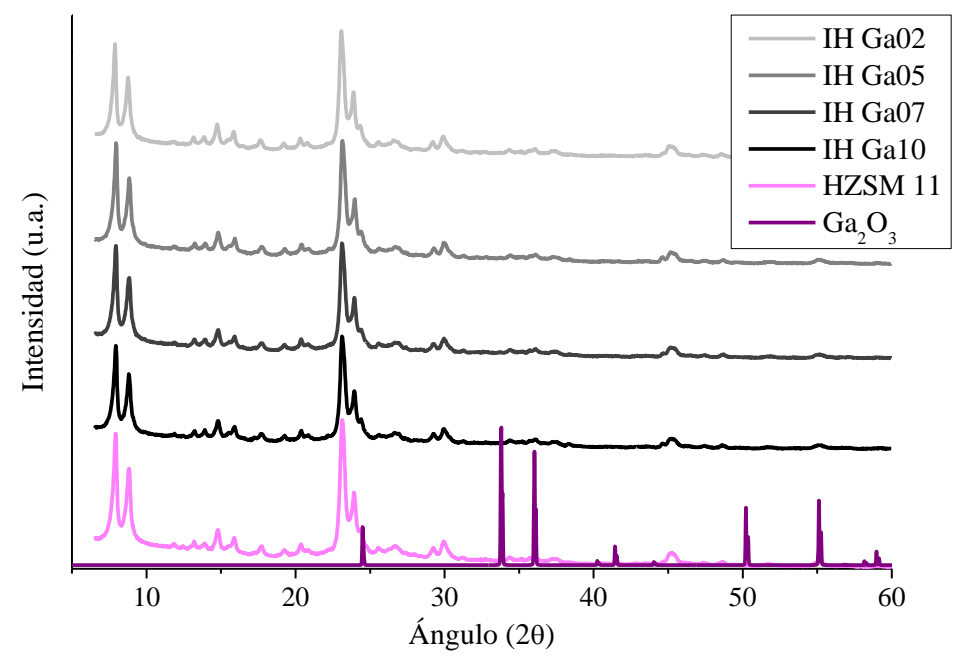

Figura 1. Patrones difracción de XRD de las zeolitas Ga-ZSM-11

Tabla 1. Caracterización Fisicoquímica

\begin{tabular}{|c|c|c|c|c|c|}
\hline Catalizador & $\begin{array}{c}\text { Cristalinidad } \\
\text { Relativa }\end{array}$ & Área Superficial & Acidez de Lewis & Acidez Total & $L / B^{b}$ \\
\hline & $\mathrm{XRD}$ & BET & & & \\
\hline & {$[\%]$} & {$\left[\mathrm{m}^{2} / \mathrm{g}\right]$} & {$[\mu \mathrm{mol} / \mathrm{g}]$} & {$[\mu \mathrm{mol} / \mathrm{g}]$} & \\
\hline H ZMS-11 & 100 & 361 & 7,54 & 76,33 & 0,11 \\
\hline IH Ga02 & 100 & 464 & 25,58 & 79,04 & 0,48 \\
\hline IH Ga05 & 100 & 367 & 25,23 & 67,85 & 0,59 \\
\hline IH Ga07 & 94 & 362 & 24,64 & 68,81 & 0,56 \\
\hline IH Ga10 & 90 & 352 & 19,21 & 54,83 & 0,54 \\
\hline
\end{tabular}

a Sitios ácidos totales determinados desde el espectro de desorción de piridina a $400^{\circ} \mathrm{C}$,

b L/B (relación sitios ácidos de Lewis y Brönsted). 
La naturaleza y fuerza de los sitios ácidos presentes en las muestras se investigó por FTIR de piridina adsorbida a temperatura ambiente, y desorbida a $250^{\circ} \mathrm{C}, 350^{\circ} \mathrm{C}$ y $400^{\circ} \mathrm{C}$. En la Figura 2 se presentaron los espectros de las muestras con distintos contenidos de galio desorbidas a $400^{\circ} \mathrm{C}$ temperatura que da cuenta de la fuerza de los sitios ácidos. El pico a $1445 \mathrm{~cm}^{-1}$ se asocia a los sitios de Lewis, y el de $1545 \mathrm{~cm}^{-1}$ con la acidez de Brönsted, siendo esta última superior en todos los casos. En la Tabla 1 se presenta la cuantificación de sitios ácidos totales y la relación entre sitios ácidos de Lewis y Brönsted. La cuantificación de los sitios (Emeis, 1993) muestra un aumento en todos los casos de sitios ácidos de Lewis en comparación con la matriz de partida (H-ZSM-11) y se observa una vez más el decrecimiento de la cantidad de estos sitios ácidos a medida que aumenta el contenido de galio incorporado en la matriz zeolítica.

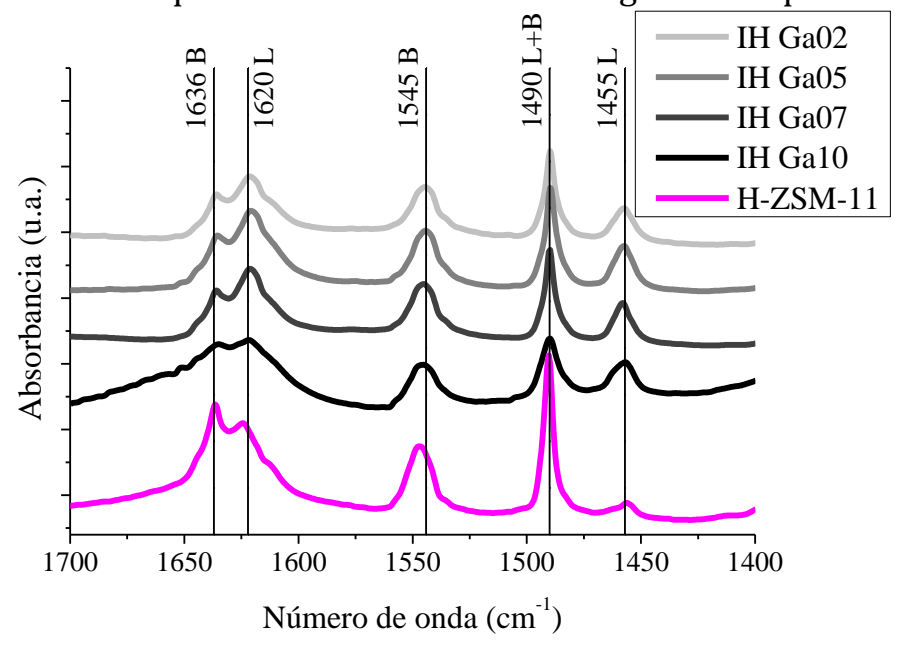

Figura 2. Espectros FTIR de piridina desorbida a $400^{\circ} \mathrm{C}$.

Se obtuvo información adicional sobre la naturaleza y la ubicación de las especies de galio en las zeolitas impregnadas por medio del análisis de reducción a temperatura programada TPR. El galio puede estar presente como especies de óxido o hidróxido depositadas en los poros o en la superficie externa de los cristales de zeolita o puede ocupar posiciones catiónicas en los canales de zeolita. Estas especies de Ga deberían tener diferentes propiedades redox dependiendo de su naturaleza y ambiente.

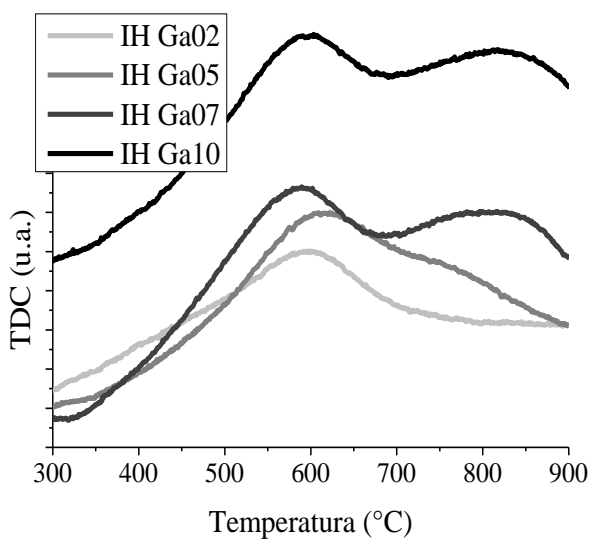

Figura 3. Espectros de Reducción a temperatura Programada

La reducción de las zeolitas Ga-ZSM-11, comienza a aproximadamente $580^{\circ} \mathrm{C}$ y alcanza un máximo alrededor de $740^{\circ} \mathrm{C}$. La Figura 3 muestra las curvas TPR observadas para las cuatro muestras impregnadas que difieren en su contenido de Ga. Se observan dos picos de reducción distintos: uno estrecho e intenso a $590^{\circ} \mathrm{C}$ cuya intensidad aumenta con el contenido de Ga, y uno amplio a 800-830 cuya intensidad se incrementa casi continuamente con la carga de Ga (en particular, en las muestras con un contenido del 7 y el 10\% p/p). En consecuencia, el último pico se asigna a partículas grandes en masa de $\mathrm{Ga}_{2} \mathrm{O}_{3}$ separadas o apoyadas en la matriz de la zeolita. El pico principal a $600^{\circ} \mathrm{C}$ se atribuye a especies de $\mathrm{Ga}$ bien dispersas, como pequeñas partículas de $\mathrm{Ga}_{2} \mathrm{O}_{3}$ o especies $\mathrm{GaO}^{+}$que interactúan con la zeolita. La determinación cuantitativa del consumo total de $\mathrm{H}_{2}$ muestra que $\mathrm{Ga}_{3}{ }^{+}$se reduce casi totalmente a $\mathrm{Ga}^{+}$en todos los casos. 


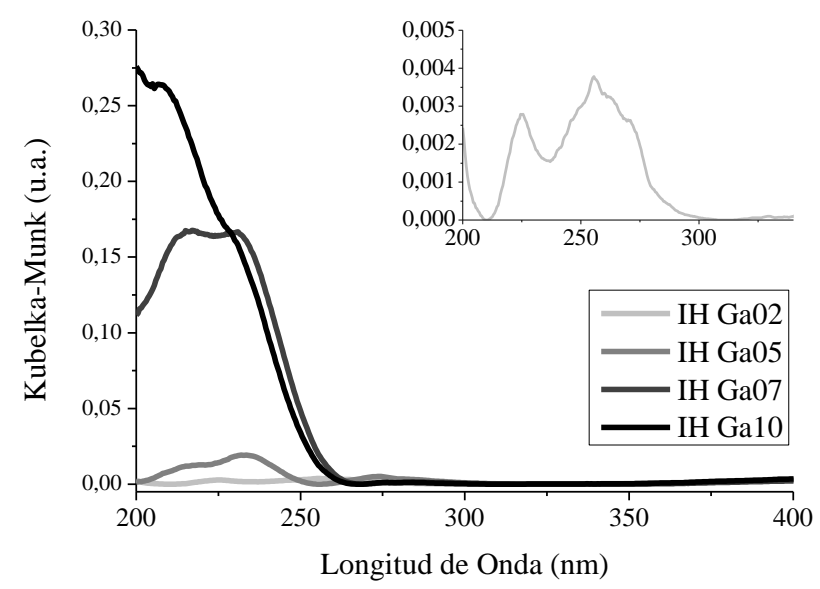

Figura 4. Espectros de DR UV-vis

La Figura 4 muestra los espectros DR-UV visibles de las muestras. La muestra con la menor cantidad de carga, IH $\mathrm{GaO}_{2}$, exhibió dos bandas anchas débiles a aproximadamente 225 y $255 \mathrm{~nm}$. Con el aumento de la carga de Ga (5 y $7 \%$ $\mathrm{p} / \mathrm{p}$ ), las bandas de absorción se corren a menores longitudes de onda y se centran a aproximadamente 215 y $235 \mathrm{~nm}$. Para IH Ga10, las bandas se hacen más pronunciadas y se manifestaron a menores longitudes de onda. Estos hallazgos indican que la estructura y el estado electrónico de las especies de galio difieren claramente entre las muestras de carga baja y alta. La banda fuerte a $235 \mathrm{~nm}$ puede adjudicarse a la absorción de nanopartículas de óxido de galio. Las bandas centradas a 215 y $240 \mathrm{~nm}$ pueden considerarse características de las especies de óxido de galio altamente dispersas en el soporte de zeolita [10]. Las especies de óxido de galio altamente dispersas exhibirían una banda de absorción a $240 \mathrm{~nm}$. Por otro lado, la banda a $215 \mathrm{~nm}$ se originaría a partir de nanopartículas de óxido de galio muy pequeñas (por ejemplo, 2-5 nm).

\section{Isomerización de DHA a ácido láctico}

Para el estudio de la obtención de ácido láctico a partir de la isomerización catalítica de dihidroxiacetona (DHA) en agua, se analizó el efecto de la temperatura, así como el porcentaje de catión efectivamente incorporado en la matriz zeolítica en la conversión del reactivo y en el rendimiento al producto de interés (Ácido Láctico).

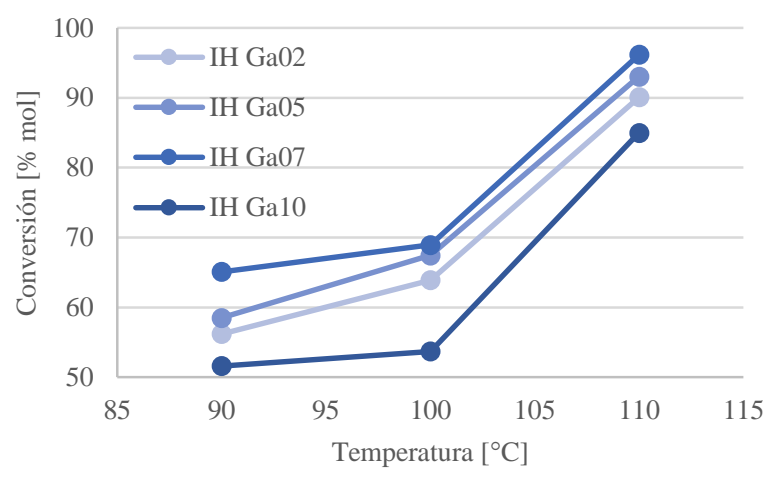

Figura 5. Conversión (mol\%) de dihidroxiacetona (DHA).

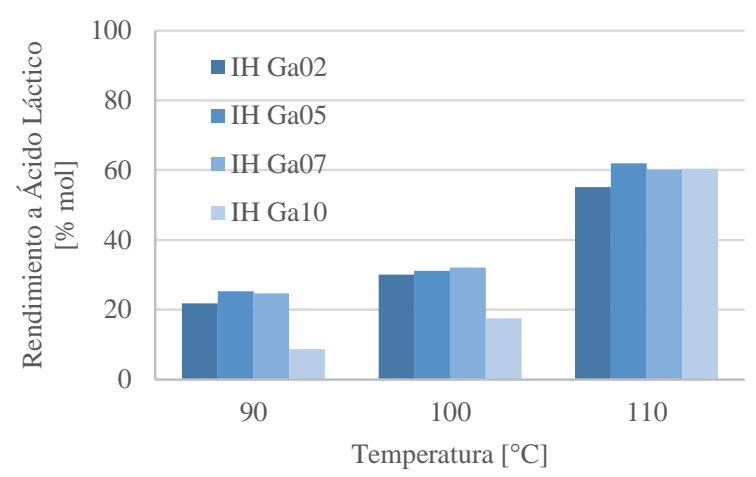

Figura 6. Rendimientos a los principales productos obtenidos.

En la Figura 5 se pueden observar las conversiones obtenidas de DHA empleando las Ga-Zeolitas. El porcentaje de conversión en todos los casos supera el $50 \%$ en mol aumentando con el incremento de la temperatura y el contenido de galio (obteniéndose cerca del $100 \%$ al emplear la muestra IH Ga07 y $110^{\circ} \mathrm{C}$ ). El catalizador con un contenido del $10 \%$ presenta los valores de conversión más bajos, este resultado puede adjudicarse a la presencia de aglomerados de $\mathrm{Ga}_{2} \mathrm{O}_{3}$, lo que se condice con los resultados de caracterización reportados en las secciones anteriores.

El principal producto obtenido en todos los casos y condiciones fue el ácido láctico, logrando un mayor rendimiento a $110^{\circ} \mathrm{C}$, para todas las cargas de metal empleadas, observándose un mejor desempeño en el al emplear el catalizador IH Ga05 con un rendimiento superior al 60\%. El rendimiento a productos secundarios no superó en ningún caso el $13 \%$. 
La naturaleza del catión efectivamente incorporado tiene un rol fundamental en los tipos de sitios ácidos presentes en el material, Brönsted o Lewis, lo que se relaciona directamente con la obtención de ácido láctico. Así el mayor valor de relación L/B concuerda con el material que presentó el mejor rendimiento. Se infiere entonces que la isomerización de DHA hacia el producto de interés estaría favorecida por a la presencia de galio, el contenido de metal en la matriz, la naturaleza y ubicación de las especies de galio efectivamente incorporadas, la cristalinidad de las mismas y el tipo de acidez.

\section{Conclusiones}

Las zeolitas modificadas con Ga se prepararon por impregnación por humedad incipiente. Las mismas fueron posteriormente empleadas en la reacción de isomerización de DHA a ácido láctico.

La caracterización de los materiales confirmó la estructura tipo ZSM 11 para todas las muestras. Por el método BET se analizó el área superficial y se encontró que la misma disminuye al aumentar el contenido de Ga incorporado. El análisis de sitios ácidos mostró una vez más el decrecimiento de la cantidad de sitios ácidos de Lewis a medida que aumenta el contenido de galio incorporado en la matriz zeolítica obteniéndose una relación L/B máxima con la muestra IH Ga05. El estudio por TPR señaló la reducción de las especies superficiales vecinas a los enlaces Ga-O y la reducción de $\mathrm{Ga}^{+3} \mathrm{a} \mathrm{Ga}^{+}$. El análisis de reflectancia difusa de UV-visible indicó la dispersión de los posibles aglomerados de óxidos de galio, aumentando la cantidad de los mismos con un aumento del contenido de metal en la matriz.

Los mayores rendimientos a ácido láctico, se obtuvieron cuando se emplearon Ga-ZSM-11 con una carga del 5\%p/p del catión metálico. La reacción que fue catalizada por el material obtenido por tratamiento alcalino exhibió un rendimiento superior al $60 \% \mathrm{p} / \mathrm{p}$ con una temperatura de reacción de $110^{\circ} \mathrm{C}$, siendo está la temperatura óptima en todos los casos puesto que a su vez se alcanza una mayor selectividad al producto de interés.

\section{Referencias}

Chu, P. (1973). United States Patent Office. https://doi.org/10.1145/178951.178972

Dapsens, P. Y., Kusema, B. T., Mondelli, C., \& Pérez-Ramírez, J. (2014). Gallium-modified zeolites for the selective conversion of bio-based dihydroxyacetone into C1-C4 alkyl lactates. Journal of Molecular Catalysis A: Chemical, 388389, 141-147. https://doi.org/10.1016/j.molcata.2013.09.032

Dapsens, P. Y., Mondelli, C., \& Pérez-Ramírez, J. (2013). Highly selective lewis acid sites in desilicated MFI zeolites for dihydroxyacetone isomerization to lactic acid. ChemSusChem, 6(5), 831-839.

https://doi.org/10.1002/cssc.201200703

Emeis, C. A. (1993). Determination of integrated molar extinction coefficients for infrared absorption bands of pyridine adsorbed on solid acid catalysts. In Journal of Catalysis (Vol. 141, Issue 2, pp. 347-354).

https://doi.org/10.1006/jcat.1993.1145

Ennaert, T., Schutyser, W., Dijkmans, J., Dusselier, M., \& Sels, B. F. (2016). Conversion of Biomass to Chemicals: The Catalytic Role of Zeolites. In Zeolites and Zeolite-like Materials. Elsevier B.V. https://doi.org/10.1016/B978-0-44463506-8.00010-0

Hayashi, Y., \& Sasaki, Y. (2005). Tin-catalyzed conversion of trioses to alkyl lactates in alcohol solution. Chemical Communications, 21, 2716-2718. https://doi.org/10.1039/b501964h

Pescarmona, P. P., Janssen, K. P. F., Stroobants, C., Molle, B., Paul, J. S., Jacobs, P. A., \& Sels, B. F. (2010). A highthroughput experimentation study of the synthesis of lactates with solid acid catalysts. Topics in Catalysis, 53(1-2), 77-85. https://doi.org/10.1007/s11244-009-9433-8 